\title{
半干旱草原土壤呼吸组分区分与菌根呼吸的贡献
}

\author{
李伟晶 1,2 陈世苹 $1^{*}$ 张兵伟 1,3 谭星儒 1,2 王珊珊 1,2 游翠 海 $1,2^{1,2}$ \\ ${ }^{1}$ 中国科学院植物研究所植被与环境变化国家重点实验室, 北京 $100093 ;{ }^{2}$ 中国科学院大学资源与环境学院, 北京 $100049 ;{ }^{3}$ 中山大学有害生物控制与 \\ 资源利用国家重点实验室, 中山大学生命科学学院, 广州 510275
}

摘 要 土壤呼吸组分的区分对于理解地下碳循环过程非常重要。而菌根真菌在地下碳循环过程中扮演着重要的角色, 但是 有关菌根呼吸在草原生态系统中的研究相对较少。该研究在内蒙古半干早草原应用深浅环网孔法, 结合浅环、深环(排除根 系)和一个带有 $40 \mu \mathrm{m}$ 孔径窗口的土壤环(排除根系但是有菌根菌丝体)将根和菌丝物理分离, 来区分不同的呼吸组分。结果表 明: 异养呼吸对总呼吸的贡献比例为 $51 \%$, 根呼吸的贡献比例为 $26 \%$, 菌根呼吸的贡献比例为 $23 \%$, 菌根呼吸的比例 3 年变化 范围为 $21 \%-26 \%$ 。与国内外研究相比, 此方法提供了一个相对稳定的菌根呼吸测量精度范围, 在草原生态系统中切实可行。 对菌根呼吸的准确定量将有助于预测草原生态系统土壤碳释放过程对未来气候变化的响应。

关键词 深浅环网孔法; 异养呼吸; 自养呼吸; 菌根呼吸; 半干旱草原

李伟晶, 陈世苹, 张兵伟, 谭星儒, 王珊珊, 游翠海 (2018). 半干旱草原土壤呼吸组分区分与菌根呼吸的贡献. 植物生态学报, 42, 850-862. DOI: $10.17521 /$ cjpe. 2018.0068

\section{Partitioning of soil respiration components and evaluating the mycorrhizal contribution to soil respiration in a semiarid grassland}

\begin{abstract}
LI Wei-Jing ${ }^{1,2}$, CHEN Shi-Ping ${ }^{*}{ }^{*}$, ZHANG Bing-Wei ${ }^{1,3}$, TAN Xing-Ru ${ }^{1,2}$, WANG Shan-Shan ${ }^{1,2}$, and YOU Cui-Hai ${ }^{1,2}$
${ }^{1}$ State Key Laboratory of Vegetation and Environmental Change, Institute of Botany, Chinese Academy of Sciences, Beijing 100093, China; ${ }^{2}$ College of Resources and Environment, University of Chinese Academy of Sciences, Beijing 100049, China; and ${ }^{3}$ State Key Laboratory of Biocontrol and School of Life Sciences, Sun Yat-sen University, Guangzhou 510275, China
\end{abstract}

\section{Abstract}

Aims Soil respiration component partitioning is pivotal to understand the belowground carbon (C) cycle. Mycorrhizal fungi have been proven to play an important role in the soil $\mathrm{C}$ turnover, but only a few studies have been conducted to quantify the contribution of mycorrhizal respiration to total soil respiration in grassland ecosystems.

Methods The mini-trenching mesh method was applied to partition soil respiration components of a semi-arid grassland in Inner Mongolia. A shallow collar (measuring soil total respiration), a deep collar (excluding roots and mycorrhizal hypahe) and a deep collar with $40 \mu \mathrm{m}$ pore mesh window (excluding roots but not mycorrhizal hyphae) were installed in each plot. Soil respiration rate of each collar was measured every two weeks during the growing season from 2014 to 2016. The differences in the rate of soil respiration among different type of collars were used to partition the components of soil respiration.

Important findings The results showed that the contribution of heterotrophic, root and mycorrhizal respiration to total soil respiration was $49 \%, 28 \%$, and $23 \%$, respectively. Across the three years, the proportion of mycorrhizal respiration varied from $21 \%-26 \%$, which is comparable with the results reported by other studies recently. Our results demonstrated that the mini-trenching mesh method is a suitable method for separating mycorrhizal respiration component in grassland ecosystems. Evaluating the contribution of mycorrhizal respiration to total soil respiration is very important for predicting the responses of soil carbon release to future climate change.

Key words the mini-trenching mesh method; heterotrophic respiration; autotrophic respiration; mycorrhizal respiration; semiarid grassland

Li WJ, Chen SP, Zhang BW, Tan XR, Wang SS, You CH (2018). Partitioning of soil respiration components and evaluating the mycorrhizal contribution to soil respiration in a semiarid grassland. Chinese Journal of Plant Ecology, 42, 850-862. DOI: $10.17521 /$ cjpe. 2018.0068

收稿日期Received: 2018-03-29 接受日期Accepted: 2018-05-29

基金项目: 国家自然科学基金(41773084)和国家重点研发计划(2017YFA0604801)。Supported by the National Natural Science Foundation of China (41773084), and the National Program on Key Research Project of China (2017YFA0604801).

* 通信作者Corresponding author (spchen@ibcas.ac.cn) 
土壤呼吸是指由土壤产生并向大气中释放 $\mathrm{CO}_{2}$ 的过程。由于从土壤表面释放的 $\mathrm{CO}_{2}$ 有多种来源, 每 个组分都包括不同的生物学和生态学过程, 对环境 变化的响应可能也不同, 所以精确区分土壤呼吸的 各种组分对于理解土壤呼吸机制本身及其对环境变 化的响应是很关键的(骆亦其和周旭辉, 2006)。土壤 呼吸可分为自养呼吸(根呼吸)和异养呼吸(微生物呼 吸和土壤动物呼吸)。严格意义上的自养呼吸只包括 根呼吸, 但由于实际研究中很难将根呼吸和根际微 生物呼吸区分开, 并且它们的分解底物均来自于植 物的光合作用, 所以通常把根呼吸和根际微生物呼 吸划分为自养呼吸, 而共生的菌根是根际微生物的 重要组成部分, 因此菌根真菌呼吸属于自养呼吸 (Hanson et al., 2000; Heinemeyer et al., 2006; Slavíková et al., 2017), 而异养呼吸主要指土壤有机 质的分解产生的呼吸组分。异养呼吸和自养呼吸都 有各自不同的驱动因素和环境限制因子, 因此区分 土壤呼吸组分对于更好地理解和预测它们各自的动 态变化是非常重要的(Neumann \& Matzner, 2014)。

已经有一些研究在不同的生态系统采用不同的 方法区分土壤呼吸组分以及定量每个组分分别对土 壤总呼吸的贡献比例。一些方法是直接测定的, 比 如离体根法: 直接测定所获得的活根的 $\mathrm{CO}_{2}$ 释放量 (Rakonczay et al., 1997; Li et al., 2008)和同位素法: 通过在植物体内添加 $\mathrm{C}$ 的示踪物来实现(Andrews et al., 1999; Hanson et al., 2000; Kuzyakov \& Larionova, 2005), 还有一些间接测定方法, 包括壕沟法(Lee et al., 2003), 环割法: 环剥植物韧皮部, 阻断碳水化 合物向根系的输入, 从而阻止根呼吸(Högberg et al., 2001; Bhupinderipal-Singh et al., 2003), 回归法(Xu et al., 2001; Rodeghiero \& Cescatti, 2006; Shi \& Geng, 2014)等。而最常用的区分自养和异养呼吸组分的方 法是壕沟法(Fisher \& Gosz, 1986), 后来在一些森 林、草原和农田生态系统中, 研究者将此方法进一 步改进, 结合网孔膜的应用, 阻止植物根系在环内 生长但允许菌根真菌的菌丝长入, 成功地估算了菌 根呼吸的大小(Heinemeyer et al., 2007; Moyano et al., 2008; Tomè et al., 2016)。

菌根真菌作为生态系统重要的功能群之一, 在 未受干扰的土壤中, 构成了植物根际区微生物的主 体, 其生物量约占微生物总量的 70\% (朱教君等, 2003)。而在自然界中分布最为广泛的菌根类型是从
枝菌根( $\mathrm{AM}), \mathrm{AM}$ 菌根是由球囊菌门真菌与植物形 成的互惠共生体, $\mathrm{AM}$ 真菌能与 $80 \%$ 以上的陆地植物 形成共生关系, 尤其与绝大多数的草本植物(Smith \& Read, 2008; Kernaghan, 2013)。由于AM真菌的菌 丝网络非常发达, 在每克土壤中能够延伸到 $30 \mathrm{~m}$ (Cavagnaro et al., 2005; Wilson et al., 2009), 并以 $0.7-1.0 \mathrm{~m} \cdot \mathrm{d}^{-1}$ 的速率生长着, 通过菌丝网络在土壤 中的延伸, 菌根真菌为植物提供了广泛的碳通量流 动和营养循环的路径(Leake et al., 2004), 因此菌根 真菌在地下碳循环过程中发挥着重要的作用(Talbot et al., 2008)。目前的研究已经发现植物光合固定的 碳有4\%-26\%分配至AM真菌(Jakobsen \& Rosendahl, 1990; Drigo et al., 2010), 但是关于菌根真菌在土壤 碳释放量方面所做贡献的了解非常有限。已有的关 于菌根呼吸定量研究的工作多集中在森林生态系统 中(Rygiewicz \& Anderson, 1994; Heinemeyer et al., 2007; Nottingham et al., 2010; Neumann \& Matzner, 2014; Lilleskov, 2017), 而在草原基于菌根呼吸组分 的原位定量研究还十分缺乏(Heinemeyer et al., 2012; Balogh et al., 2016)。在国内关于土壤呼吸组分区分 的研究主要集中在自养和异养呼吸组分的区分 (Wang \& Yang, 2007; Yan et al., 2010; Zhang et al., 2013), 目前关于菌根呼吸组分区分的研究还未见 报道。

本研究以内蒙古半干旱草原生态系统为研究对 象, 采用Johnson等(2001)所提出的在土壤环上打开 窗口安装一定规格的网孔膜排除根系的方法，区分 根呼吸、菌根呼吸和异养呼吸这 3 个土壤呼吸主要组 分, 并通过连续 3 年土壤呼吸各组分季节和年际变 化的测定，定量它们对土壤总呼吸贡献的比例。

\section{1 材料和方法}

\section{1 试验样地概况}

本研究样地位于内蒙古自治区锡林浩特市西乌 珠穆沈旗的半干早草原 $\left(44.37^{\circ} \mathrm{N}, 117.58^{\circ} \mathrm{E}\right.$ ，海拔 $1148 \mathrm{~m})$ 。本地区属于中温带半干旱大陆性气候, 根 据西乌珠穆沁旗气象站1955-2011年的监测数据(离 本研究站点距离约 $30 \mathrm{~km})$, 该地区年平均气温 $1.5{ }^{\circ} \mathrm{C}$, 其中月平均气温最高发生在 7 月为 $19.8{ }^{\circ} \mathrm{C}$, 最低月平均气温发生在 1 月为 $-19.0{ }^{\circ} \mathrm{C}$ 。年降水量为 $333 \mathrm{~mm}$, 其中约 $87 \%$ 的降水发生在生长季的 5-9月 份。实验期间(2014-2016年生长季)自然降水量分别 
为 $281 、 290$ 和 $271 \mathrm{~mm}$ 。本研究样地于 2011 年 8 月开 始围封, 围封前为打草场, 每年进行一次打草。该地 区天然植被以典型草原为主, 土壤类型为栗钙土, 植物种类主要由多年生 $\mathrm{C}_{3}$ 禾草及杂类草构成。优势 植物有大针茅(Stipa grandis)、羊草(Leymus chinensis) 和知母(Anemarrhena asphodeloides)等。

\section{2 研究方法}

本实验样方于 2012 年 5 月建立，每个样方大小 为 $3 \mathrm{~m} \times 4 \mathrm{~m}$, 样方间隔为 $1 \mathrm{~m}$, 总共包含 4 个重复。 在每个样方中嵌入 3 个不同的土壤环, 用于土壤呼 吸组分的区分, 分别为浅环、深环和菌丝环(图1)。 它们均是直径为 $11 \mathrm{~cm}$ 的聚氯乙烯 $(\mathrm{PVC})$ 环, 浅环的 高度是 $6 \mathrm{~cm}$, 深环和菌丝环高度是 $40 \mathrm{~cm}$ 。在实验测 定前 1 年将土壤环砸入土壤中, 高出地表 $3 \mathrm{~cm}$ 左右, 并要注意及时清理土壤环中的绿色植物, 以消除地 上植物呼吸部分对土壤呼吸测定的干扰。浅环用于 测定土壤总呼吸 $\left(S R_{\mathrm{tot}}\right)$ 。研究表明, 本区域大部分 (约 $80 \%$ 以上)的植物根系分布在土壤 $30 \mathrm{~cm}$ 深度范围 内(马文红等, 2008), 因而本实验中的深环能够隔断
大部分的植物根系并阻断新根长入土壤环内, 因而 采用深环测定的土壤呼吸是土壤异养呼吸组分 $\left(S R_{\mathrm{h}}\right)$ 。菌丝环的高度和深环相同, 在菌丝环距边缘5 $\mathrm{cm}$ 处设置 3 个 $15 \mathrm{~cm} \times 5 \mathrm{~cm}$ 大小的窗口，窗口上贴上 孔径为 $40 \mu \mathrm{m}$ 的尼龙网。有研究表明菌丝直径在 10-20 $\mu \mathrm{m}$ 之间(Friese \& Allen, 1991), 这样尼龙网只 允许菌丝通过而植物根系不能通过。因而菌丝环测 得的土壤呼吸是土壤异养呼吸与菌丝呼吸 $\left(S R_{\mathrm{my}}\right)$ 之 和, 浅环土壤呼吸测定值用 $S R_{\mathrm{sc}}$ 表示, 深环土壤呼 吸测定值用 $S R_{\mathrm{dc}}$ 表示, 菌丝环土壤呼吸测定值用 $S R_{\mathrm{hc}}$ 表示, 自养呼吸用 $S R_{\mathrm{a}}$ 表示, 根呼吸用 $S R_{\mathrm{r}}$ 表示。 然后利用公式(1)-(3)计算土壤呼吸各个组分。

$$
\begin{aligned}
& S R_{\mathrm{a}}=S R_{\mathrm{tot}}-S R_{\mathrm{h}} \\
& S R_{\mathrm{r}}=S R_{\mathrm{tot}}-\left(S R_{\mathrm{h}}+S R_{\mathrm{my}}\right) \\
& S R_{\mathrm{my}}=\left(S R_{\mathrm{h}}+S R_{\mathrm{my}}\right)-S R_{\mathrm{h}}
\end{aligned}
$$

采用便携式土壤呼吸自动测定系统(LI-8100, LI-COR, Lincoln, USA)测定土壤呼吸。测定时间为 2014-2016年生长季, 每10天一次, 每次测定在 8:00-11:00之间进行。同时进行表层土壤 $(0-10 \mathrm{~cm})$

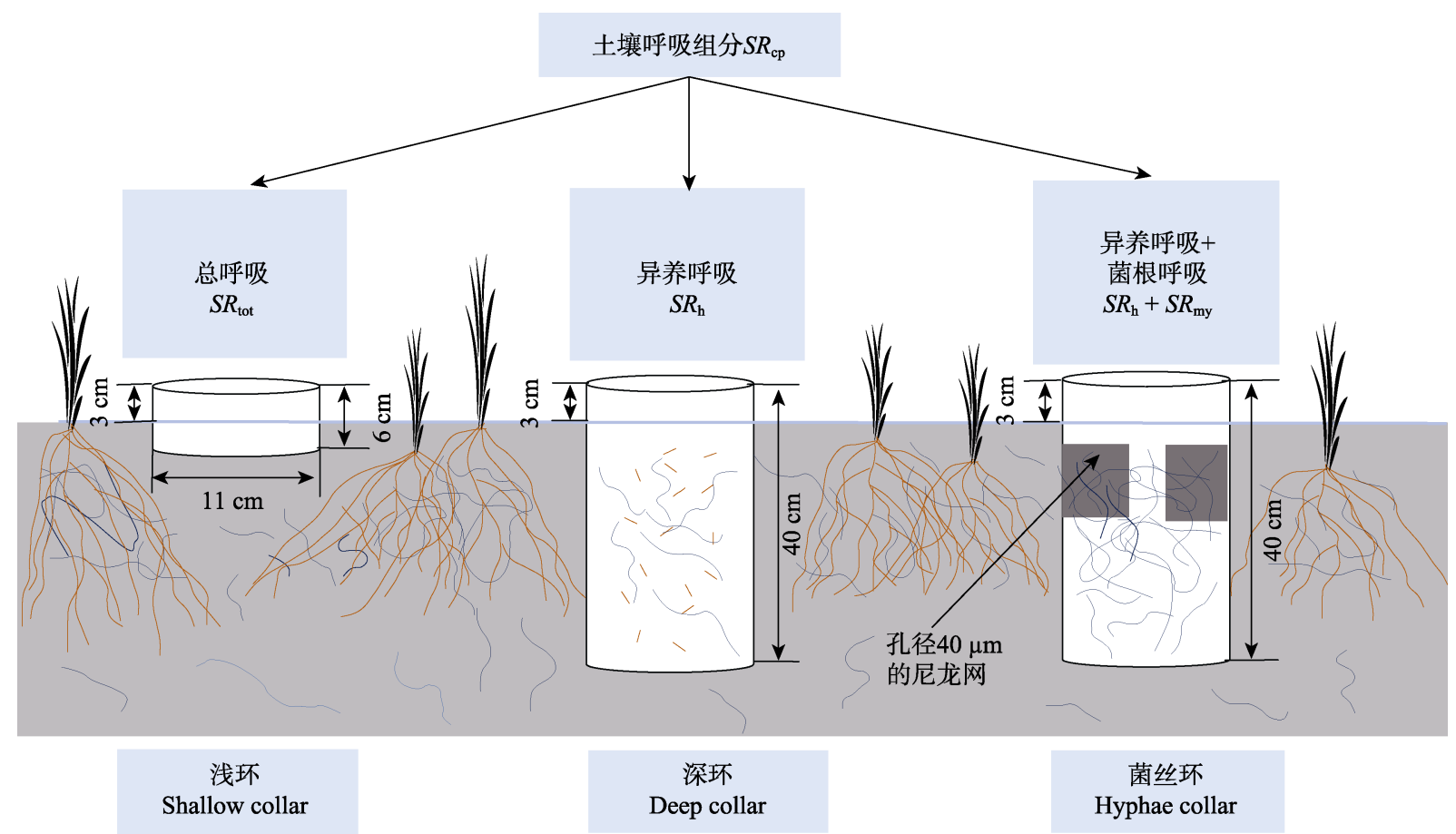

图1 改进的深浅环法区分土壤呼吸组分示意图(曲线表示菌丝体, 短线表示碎屑)。浅环用来测定土壤总呼吸, 深环用来测定 土壤异养呼吸, 菌丝环用来测定土壤异养呼吸和菌根呼吸的和, 浅环和深环测得的土壤呼吸之差为土壤自养呼吸, 浅环和菌 丝环测得的土壤呼吸之差为根呼吸，菌丝环和深环测得的土壤呼吸之差为菌根呼吸。

Fig. 1 Diagram of the modified mini-trenching method in partitioning soil respiration into its components (The curve denotes mycelium and the short line denotes detritus). The shallow and deep collars are used to determine total soil respiration $\left(S R_{\mathrm{tot}}\right)$ and its heterotrophic component $\left(S R_{\mathrm{h}}\right)$. Hyphae collar is used for the determination of soil heterotrophic respiration and hyphae respiration $\left(S R_{\mathrm{h}}+S R_{\mathrm{my}}\right)$. The difference in soil respiration between the shallow and deep collars is used to represent autotrophic soil respiration $\left(S R_{\mathrm{a}}\right)$, that between the shallow and hyphae collars is used to represent root respiration $\left(S R_{\mathrm{r}}\right)$, and that between the hyphae and deep collars is used to represent hyphae respiration $\left(S R_{\mathrm{my}}\right)$.

www.plant-ecology.com 
的含水量和土壤温度的测定。土壤含水量由便携式 TDR-300 (Spectrum Technologies, Plainfield, USA) 测定, 土壤温度由LI-8100附带的土壤热电偶探头 (LI-8100-201)测定。为了减少土壤温度和水分测定 对环内土壤造成的扰动, 深环和菌丝环的环境指标 测量仅在2014年生长季进行, 并建立其与浅环土壤 温度和水分之间的关系。2015和2016年深环和菌丝 环的土壤温度和土壤水分通过其与浅环之间的相关 关系方程计算得到。

在 2016年生长季旺季8月中旬, 分别在每个样 方的浅环、深环和菌丝环采集 $0-10 \mathrm{~cm}$ 的土壤样品, 总共有 4 个重复, 用于菌丝密度的测定。参照 Jakobsen等(1992)和Rillig等(1999)的方法进行AM真 菌菌丝的提取。具体步骤如下: 称取 $4 \mathrm{~g}$ 新鲜的土壤 样品溶于 $100 \mathrm{~mL}$ 蒸馏水中并加入 $5 \mathrm{~mL} 35 \mathrm{~g} \cdot \mathrm{L}^{-1}$ 偏磷 酸钠溶液, 振荡土壤悬浮液 $30 \mathrm{~s}$, 使混合均匀, 然后 静置 $30 \mathrm{~min}$ 将上清液过 400 目筛进行过滤。将筛上的 残留物用蒸馏水转移到 $250 \mathrm{~mL}$ 三角瓶中, 并定容至 $200 \mathrm{~mL}$ 。摇动瓶身 $5 \mathrm{~s}$ 后静置 $1 \mathrm{~min}$, 用移液器取 $2 \mathrm{~mL}$ 悬浮液进行抽滤, 使之转入 $25 \mu \mathrm{m}$ 的微孔滤膜上。将 滤膜取下(有菌丝的一面朝上)转移到载玻片上, 用 酸性品红染色 $1 \mathrm{~h}$ 后置于 200 倍显微镜下进行观察, 并根据菌丝颜色和形态区分 $\mathrm{AM}$ 真菌菌丝和非 $\mathrm{AM}$ 真菌菌丝(Miller et al., 1995)。

\section{3 数据统计分析}

运用SPSS 21.0软件对数据进行统计分析, 为了 解不同土壤呼吸环处理间是否存在显著性差异, 对 不同土壤呼吸环内的土壤温度、土壤水分和菌丝密 度进行单因素方差分析, 采用最小显著差数法 $(L S D)$ 进行多重比较。在本研究中土壤呼吸及其组分的季 节平均值是由生长季5-8月份所有测定结果平均值 得来。采用相关分析研究土壤呼吸及其组分与土壤 温度以及土壤水分之间的关系, 采用多元回归分析 构建土壤呼吸与土壤温度和土壤水分的拟和方程, 所有检验显著性水平设定为 $p<0.05$, 由统计分析 软件SAS 9.2完成。文中的图采用SigmaPlot 12.5绘 制。

\section{2 结果}

\section{1 不同土壤呼吸环对菌丝密度的影响}

方差分析结果表明, 浅环、深环和菌丝环内 $\mathrm{AM}$ 菌根真菌菌丝密度有显著差别(图 $2, p<0.05$ ), 其中
菌丝环内的菌丝密度最高, 为 $2.83 \mathrm{~m} \cdot \mathrm{g}^{-1}$ 干土, 而深 环土壤中的菌丝密度最低, 为 $0.76 \mathrm{~m} \cdot \mathrm{g}^{-1}$ 干土。

\section{2 不同土壤呼吸环对土壤温度、水分的影响}

由2014年5、6月份的浅环、深环和菌丝环的土 壤温度季节变化和方差分析结果表明: 浅环、深环 和菌丝环内的土壤温度没有显著性差异(图3A、3B, $p=0.16)$, 它们均和浅环内的土壤温度呈显著的线 性关系(图4A、4C)。不同土壤环内的土壤含水量存 在显著性差异(图3C、3D, $p<0.01$ )。与浅环相比, 深 环和菌丝环内的土壤表层含水量分别显著提高了 $38 \%$ 和 $33 \%$, 且深环和菌丝环内的土壤水分含量分 别和浅环内的水分含量呈幂指数关系 $\left(y=4.95 x^{0.50}\right.$; $y=4.71 x^{0.51}$ ), 且相关性较高, 分别为 0.77 和 0.78 (图 $4 \mathrm{~B} 、 4 \mathrm{D})$ 。

\section{3 土壤呼吸组分的区分与校正}

通过测定浅环、深环和菌丝环的土壤呼吸以及 土壤温度和含水量, 2014-2016三年的数据表明浅 环、深环和菌丝环的土壤呼吸分别与土壤温度呈幂指 数关系(图5), 与土壤含水量呈二次函数关系(图6)。

为了消除土壤环境变化对深环所测得土壤呼吸 $\left(S R_{\mathrm{dc}}\right)$ 与菌丝环所测得土壤呼吸 $\left(S R_{\mathrm{hc}}\right)$ 的影响, 本研 究采用一个幂指数-二次方程来模拟它们与土壤温 度 $\left(T_{\mathrm{s}}\right)$ 和土壤含水量 $(S W C)$ 之间的关系(Saiz et al., 2007; Yan et al., 2010)。

$$
\begin{aligned}
& S R_{\mathrm{dc}}=a_{1} \mathrm{e}^{b_{1} T_{\mathrm{s}}}\left(c_{1} S W C^{2}+d_{1} S W C\right) \\
& S R_{\mathrm{hc}}=a_{2} \mathrm{e}^{b_{2} T_{\mathrm{s}}}\left(c_{2} S W C^{2}+d_{2} S W C\right)
\end{aligned}
$$

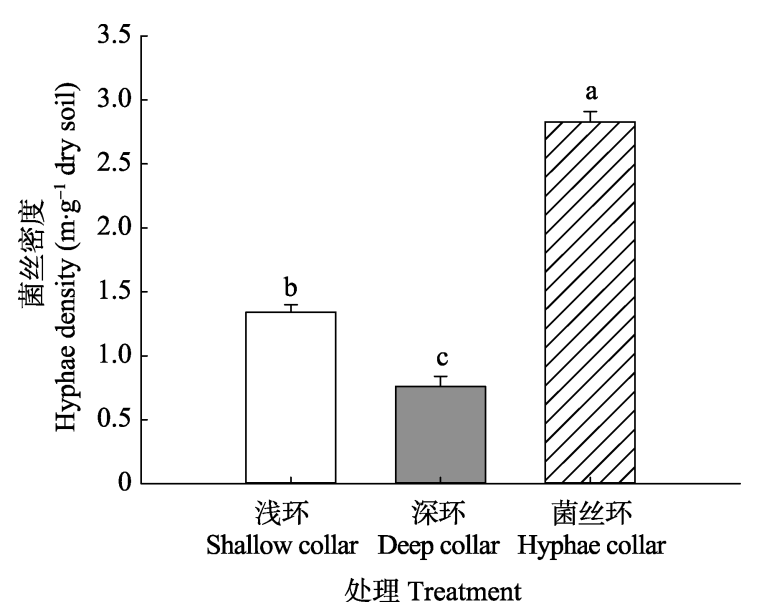

图2 不同土壤呼吸环内的菌丝密度(平均值+标准误差)。不 同小写字母表示处理间差异显著 $(p<0.05)$ 。

Fig. 2 Hyphae density under different soil respiration collars $($ mean $+S E)$. Different letters indicate significant differences among treatments $(p<0.05)$. 

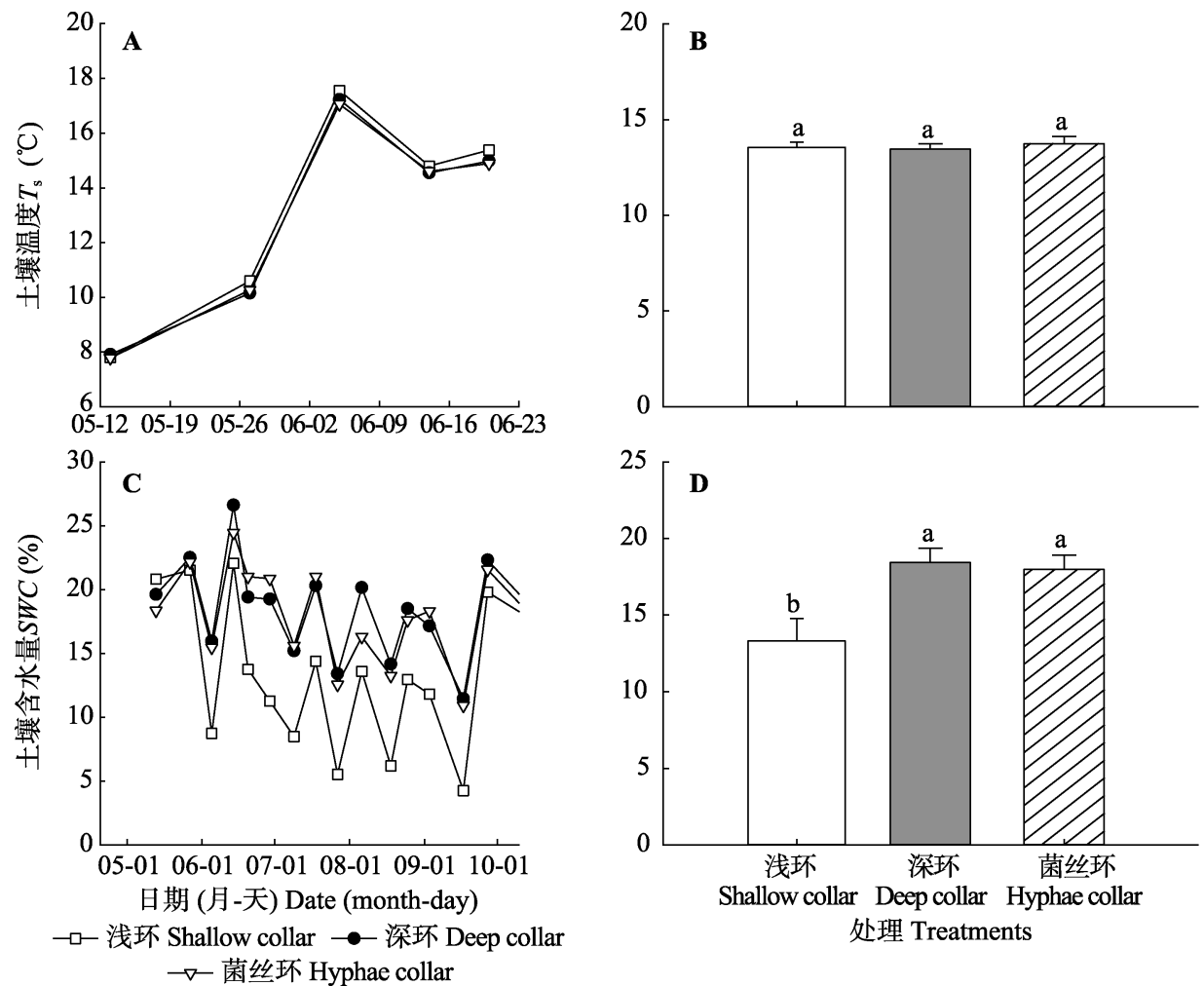

图3 2014年不同土壤呼吸环内的土壤温度 $\left(T_{\mathrm{s}}\right)$ 、土壤含水量 $(S W C)$ 的季节动态 $(\mathbf{A}, \mathbf{C})$ 和年平均值 $(\mathbf{B}, \mathbf{D})($ 平均值+标准误差)。不 同小写字母表示处理间差异显著 $(p<0.05)$ 。

Fig. 3 Seasonal variations of soil temperature $\left(T_{\mathrm{s}}\right)$, soil water content $(S W C)$ under different soil respiration collars during 2014 (A, C) and average from the year $2014(\mathbf{B}, \mathbf{D})($ mean $+S E)$. Different lowercase letters indicate significant differences among treatments $(p<0.05)$
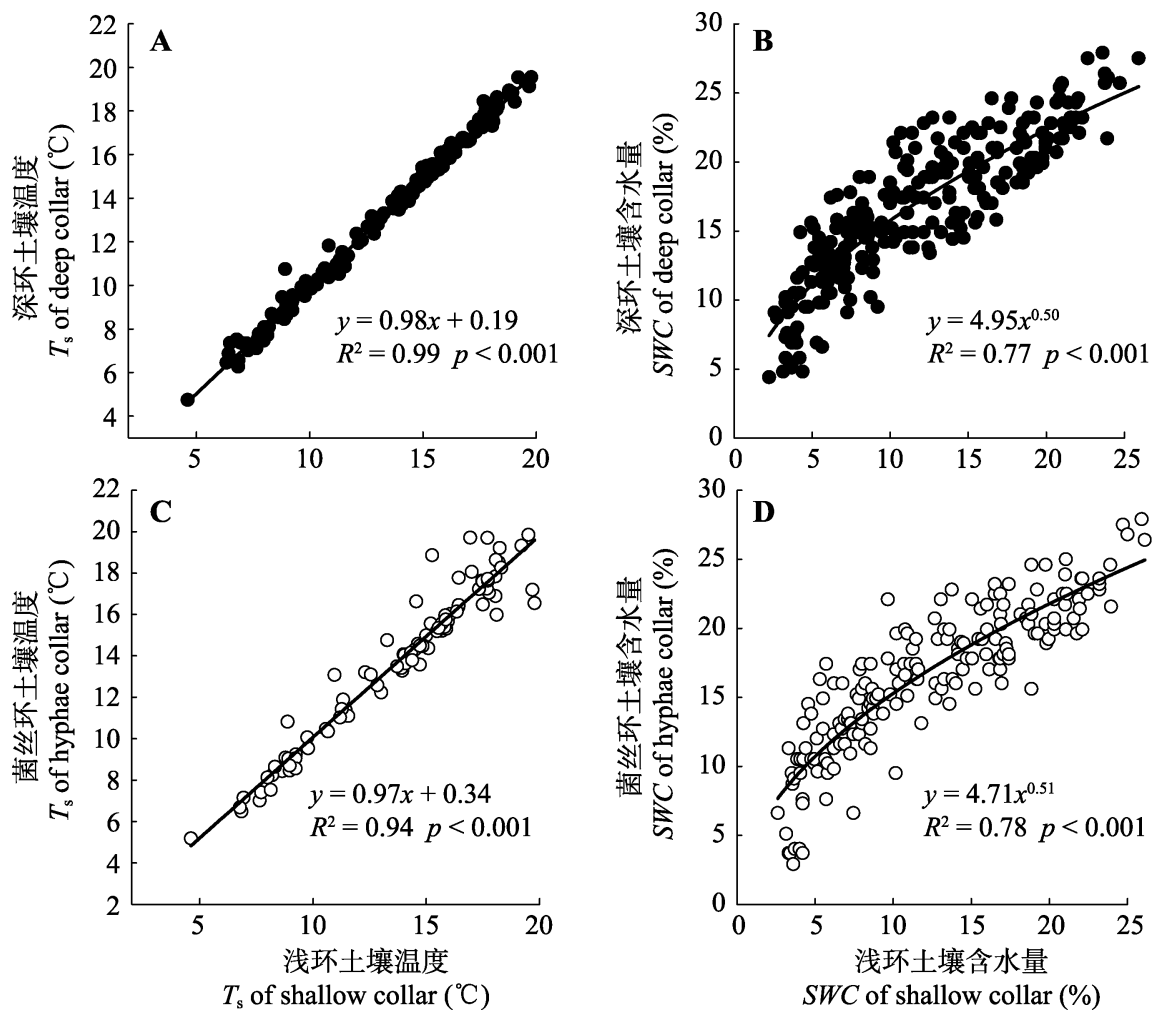

图4 不同土壤环内土壤温度之间的关系 $(A, C)$ 以及不同土壤环内土壤含水量之间的关系 $(B, D)$ 。

Fig. 4 The relationship between soil temperature $\left(T_{\mathrm{s}}\right)$ in different soil collars $(\mathbf{A}, \mathbf{C})$ and the relationship between soil water content $(S W C)$ in different soil collars $(\mathbf{B}, \mathbf{D})$.

www.plant-ecology.com 

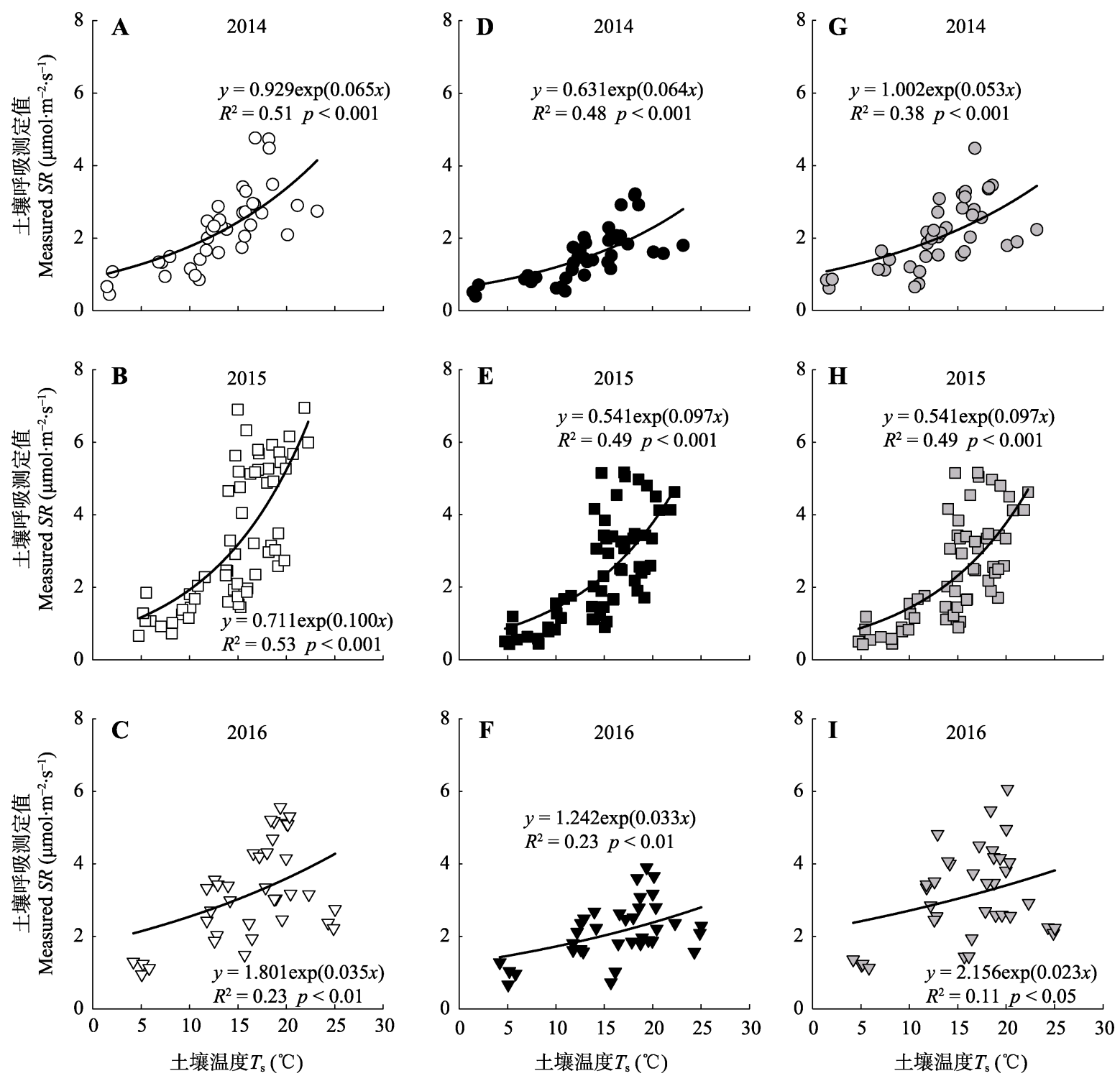

图5 2014-2016年不同土壤呼吸环测得的土壤呼吸与土壤温度之间的关系。A, B, C, 浅环; D, E, F, 深环; G, H, I, 菌丝环。 Fig. 5 The relationship between soil respiration $(S R)$ and soil temperature $\left(T_{\mathrm{s}}\right)$ in different soil respiration collars during 2014-2016. A, B, C, Shallow collar. D, E, F, Deep collar. G, H, I, Hyphae collar.

其中 $S R_{\mathrm{dc}}$ 为深环土壤呼吸测定值, $S R_{\mathrm{hc}}$ 为菌丝环土壤 呼吸测定值, $T_{\mathrm{s}}$ 为土壤温度, $S W C$ 为土壤水分。 $a_{1}-d_{1}$ 和 $a_{2}-d_{2}$ 为方程模拟出来的参数。通过这一过程得到 $S R_{\mathrm{dc}}$ 与 $T_{\mathrm{s}}$ 和 $S W C$ 之间的关系以及 $S R_{\mathrm{hc}}$ 与 $T_{\mathrm{s}}$ 和 $S W C$ 之 间的关系(表1), 然后将浅环的 $T_{\mathrm{s}}$ 和 $S W C$ 代入表1的 方程中, 即可求算出与浅环土壤环境相同的异养呼 吸组分 $S R_{\mathrm{h}}$ 。将其与浅环中测得的 $S R_{\mathrm{tot}}$ 求差, 即可得 到自养呼吸组分 $S R_{\mathrm{a}}$ 。同理, 将浅环的 $T_{\mathrm{s}}$ 和 $S W C$ 代入 菌丝环土壤呼吸拟和方程中, 即可求算出与浅环土 壤环境相同的异养呼吸 $S R_{\mathrm{h}}$ 与菌丝呼吸 $S R_{\mathrm{my}}$ 之和, 利用公式(2), 将其与浅环中测得的 $S R_{\mathrm{tot}}$ 求差, 即可 得到自养呼吸中的根系呼吸组分 $S R_{\mathrm{r}}$, 利用公式(3),
将其与深环测得的 $S R_{\mathrm{h}}$ 求差, 即可求得菌丝呼吸组 分 $S R_{\mathrm{my}}$ 。

\section{4 深环和菌丝环土壤呼吸观测值和校正值的季} 节动态

2014-2016年深环和菌丝环校正后的土壤呼吸, 与实测值相比降低了(图7)。2014年深环土壤呼吸校 正值与实测值相比平均降低了 $0.54 \mu \mathrm{mol} \cdot \mathrm{m}^{-2} \cdot \mathrm{s}^{-1}$, 菌 丝环的土壤呼吸速率平均降低了 $0.72 \mu \mathrm{mol} \cdot \mathrm{m}^{-2} \cdot \mathrm{s}^{-1}$, 2015年深环和菌丝环实测的土壤呼吸校正值与实测 值相比分别平均降低了 0.68 和 $0.78 \mu \mathrm{mol} \cdot \mathrm{m}^{-2} \cdot \mathrm{s}^{-1}$, 2016 年两者分别平均降低了 0.41 和 $0.57 \mu \mathrm{mol} \cdot \mathrm{m}^{-2} \cdot \mathrm{s}^{-1}$, 3 年中菌丝环的土壤呼吸校正值与实测值的平均差 

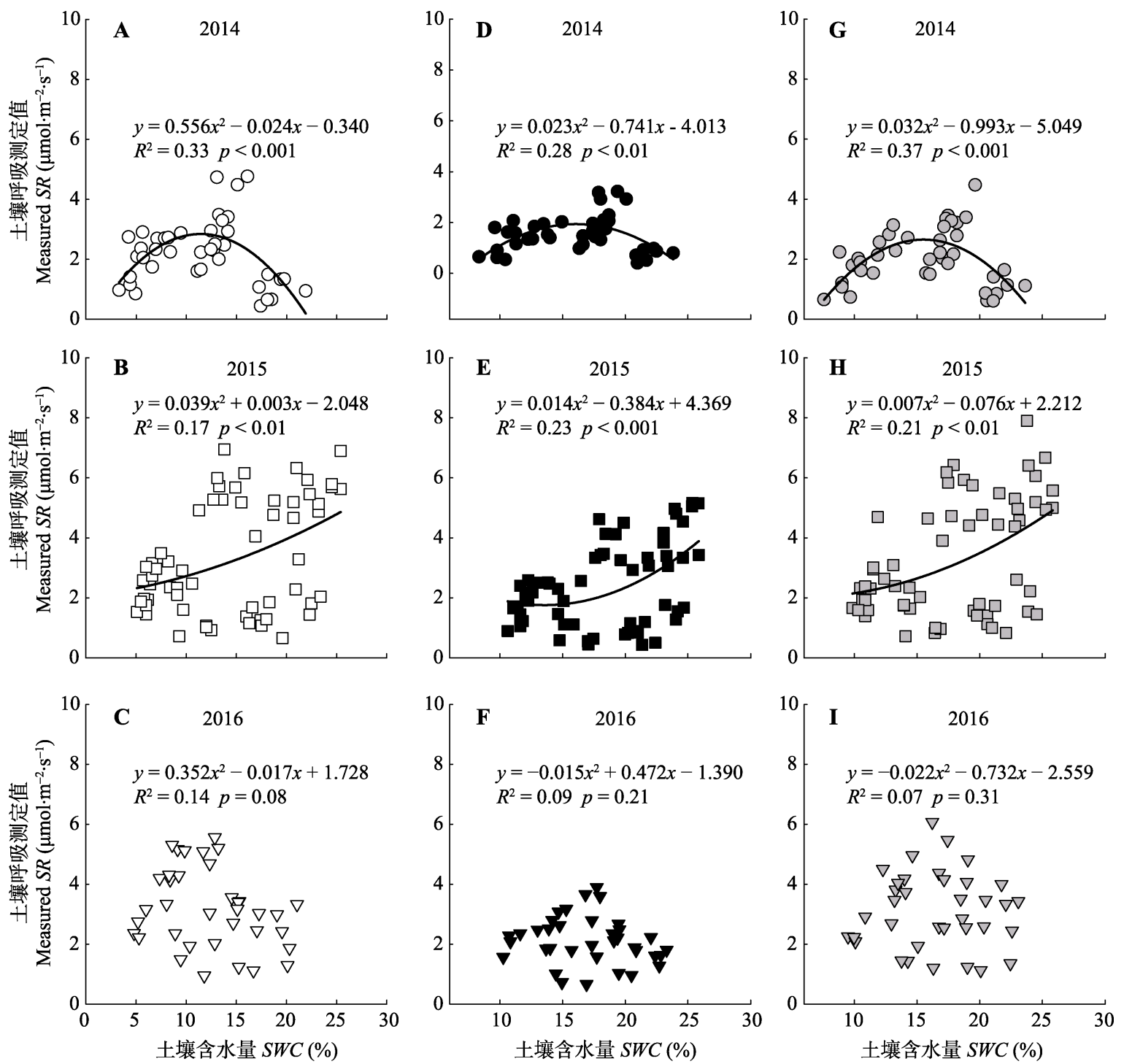

图6 2014-2016年不同土壤呼吸环测得的土壤呼吸与土壤含水量之间的关系。A, B, C, 浅环; D, E, F, 深环; G, H, I, 菌丝环。 Fig. 6 The relationship between soil respiration $(S R)$ and soil water content $(S W C)$ in different soil respiration collars during 2014-2016. A, B, C, Shallow collar. D, E, F, Deep collar. G, H, I, Hyphae collar.

表1 浅环 $\left(S R_{\mathrm{sc}}\right)$ 、深环 $\left(S R_{\mathrm{dc}}\right)$ 和菌丝环呼吸 $\left(S R_{\mathrm{hc}}\right)$ 实测值与土壤温度 $\left(T_{\mathrm{s}}\right)$ 和含水量 $(S W C)$ 拟和方程及相关参数 $\left(N, R^{2}\right.$ 和 $p$ 值 $)$

Table 1 Functions and model fit parameters $\left(N, R^{2}\right.$ and $p$-values) between measured soil respiration of shallow collar $\left(S R_{\mathrm{sc}}\right)$, deep collar ( $\left.S R_{\mathrm{dc}}\right)$, and hyphae collar $\left(S R_{\mathrm{hc}}\right)$ with soil temperature $\left(T_{\mathrm{s}}\right)$ and soil water content $(S W C)$

\begin{tabular}{|c|c|c|c|c|c|}
\hline 年份 Year & 土壤环类型 Collar type & 样本数量 $N$ & 方程 Function & $R^{2}$ & $p$ \\
\hline \multirow[t]{3}{*}{2014} & 浅环 Shallow collar & 64 & $Y=0.032 \mathrm{e}^{0.098 T_{\mathrm{s}}}\left(-0.084 S W C^{2}+2.70 S W C\right)$ & 0.82 & $<0.0001$ \\
\hline & 深环 Deep collar & 64 & $Y=0.011 \mathrm{e}^{0.110 T \mathrm{~s}}\left(0.032 S W C^{2}+1.20 S W C\right)$ & 0.75 & $<0.0001$ \\
\hline & 菌丝环 Hyphae collar & 64 & $Y=0.011 \mathrm{e}^{0.099 T \mathrm{~s}}\left(0.082 S W C^{2}+2.00 S W C\right)$ & 0.72 & $<0.0001$ \\
\hline \multirow[t]{3}{*}{2015} & 浅环 Shallow collar & 60 & $Y=0.020 \mathrm{e}^{0.109 T_{\mathrm{s}}}\left(-0.037 S W C^{2}+2.90 S W C\right)$ & 0.87 & $<0.0001$ \\
\hline & 深环 Deep collar & 60 & $Y=0.011 \mathrm{e}^{0.108 T \mathrm{~s}}\left(0.043 S W C^{2}+1.55 S W C\right)$ & 0.86 & $<0.0001$ \\
\hline & 菌丝环 Hyphae collar & 60 & $Y=0.009 \mathrm{e}^{0.113 T \mathrm{~s}}\left(0.045 S W C^{2}+2.65 S W C\right)$ & 0.89 & $<0.0001$ \\
\hline \multirow[t]{3}{*}{2016} & 浅环 Shallow collar & 36 & $Y=0.095 \mathrm{e}^{0.053 \mathrm{Ts}}\left(-0.090 S W C^{2}+2.40 S W C\right)$ & 0.44 & $<0.0001$ \\
\hline & 深环 Deep collar & 36 & $Y=0.032 \mathrm{e}^{0.049 T \mathrm{~s}}\left(-0.064 S W C^{2}+2.90 S W C\right)$ & 0.37 & $<0.0001$ \\
\hline & 菌丝环 Hyphae collar & 36 & $Y=0.061 \mathrm{e}^{0.043 T \mathrm{~s}}\left(-0.043 S W C^{2}+2.30 S W C\right)$ & 0.28 & $<0.0001$ \\
\hline
\end{tabular}



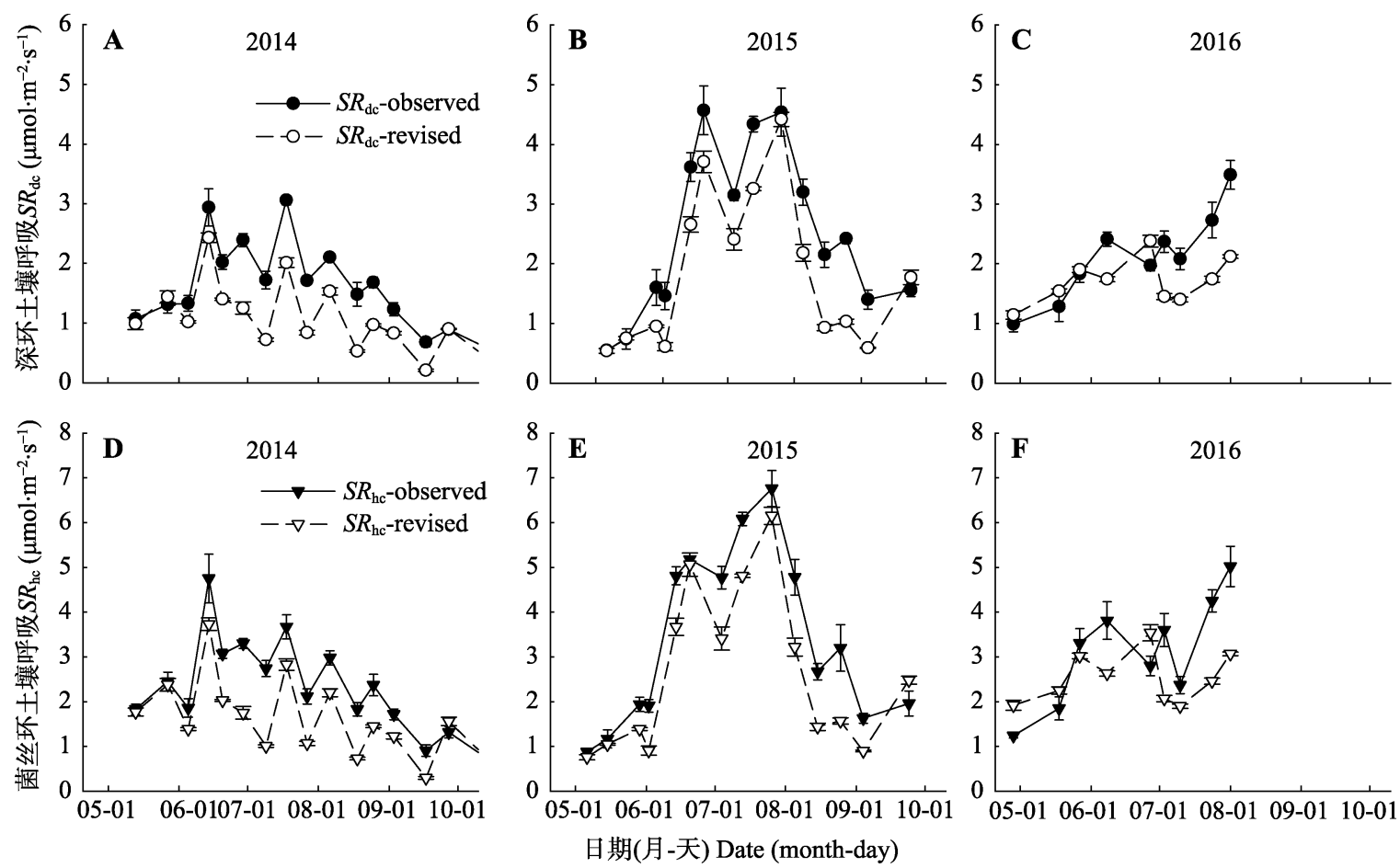

图7 2014-2016年深环和菌丝环土壤呼吸的观测值与校正值的季节动态(平均值土标准误差)。SR $R_{\mathrm{dc}}$-observed、SR $R_{\mathrm{dc}}$-revised $S R_{\mathrm{hc}}$-observed、SR $R_{\mathrm{hc}}$-revised分别表示深环土壤呼吸的观测值、深环土壤呼吸的校正值、菌丝环土壤呼吸的观测值、菌丝环土 壤呼吸的校正值。

Fig. 7 The seasonal dynamics of the observed value of soil respiration and the revised value of deep collar and hyphae collar (mean $\pm S E)$. $S R_{\mathrm{dc}}$-observed, $S R_{\mathrm{dc}}$-revised, $S R_{\mathrm{hc}}$-observed and $S R_{\mathrm{hc}}$-revised represent the observed value of soil respiration in the deep collar, the revised value of soil respiration in the deep collar, the observed value of soil respiration in the hyphae collar, and the revised value of soil respiration in the hyphae collar, respectively.

值比深环的更大, 且最大差值达 $1.95 \mu \mathrm{mol} \cdot \mathrm{m}^{-2} \cdot \mathrm{s}^{-1}$ 。

\section{5 校正后土壤呼吸各组分的季节动态}

在2014-2016年生长季中, 土壤异养呼吸和自 养呼吸以及自养组分中的根呼吸和菌根呼吸均呈现 出明显的季节变化, 其峰值一般出现在生长季旺季 的 7-8月份(图8), 最小值一般出现在生长季的初期 和末期, 在 3 年中土壤总呼吸的最大值为 $6.2 \mu \mathrm{mol}$ $\mathrm{m}^{-2} \cdot \mathrm{s}^{-1}$, 异养呼吸的最大值为 $3.71 \mu \mathrm{mol} \cdot \mathrm{m}^{-2} \cdot \mathrm{s}^{-1}$, 自 养呼吸的最大值为 $3.11 \mu \mathrm{mol} \cdot \mathrm{m}^{-2} \cdot \mathrm{s}^{-1}$, 根呼吸和菌根 呼吸的最大值分别为 2.48 和 $1.56 \mu \mathrm{mol} \cdot \mathrm{m}^{-2} \cdot \mathrm{s}^{-1}$, 菌根 呼吸的最小值为 $0.09 \mu \mathrm{mol} \cdot \mathrm{m}^{-2} \cdot \mathrm{s}^{-1}$ 。

\section{6 土壤呼吸各个组分以及所占比例的年际变化}

土壤呼吸各个组分呈现出明显的年际波动(图

$9)$, 并且在2014到2016年, 异养呼吸 $\left(S R_{\mathrm{h}}\right)$ 的变化范 围是 1.29-2.04 $\mu \mathrm{mol} \cdot \mathrm{m}^{-2} \cdot \mathrm{s}^{-1}$, 菌根呼吸 $\left(S R_{\mathrm{my}}\right)$ 的变化 范围是 $0.61-0.86 \mu \mathrm{mol} \cdot \mathrm{m}^{-2} \cdot \mathrm{s}^{-1}$, 根呼吸 $\left(S R_{\mathrm{r}}\right)$ 的变化 范围是 $0.63-0.98 \mu \mathrm{mol} \cdot \mathrm{m}^{-2} \cdot \mathrm{s}^{-1}$ 。

在2014-2016年，土壤呼吸各个组分占总呼吸 的比例呈现明显的年际变化(图9)。异养呼吸占总呼 吸的比例 $\left(S R_{\mathrm{h}} / S R_{\mathrm{tot}}\right)$ 年均值为 $51 \%$, 菌根呼吸占总呼
吸的比例 $\left(S R_{\mathrm{my}} / S R_{\mathrm{tot}}\right)$ 年均值为 $23 \%$, 根呼吸占总呼 吸的比例 $\left(S R_{\mathrm{r}} / S R_{\mathrm{tot}}\right)$ 年均值为 $26 \%$, 在 2014-2016年 菌根呼吸占自养呼吸的比例 $\left(S R_{\mathrm{my}} / S R_{\mathrm{a}}\right)$ 年均值为 $48 \%$, 根呼吸占自养呼吸的比例 $\left(S R_{\mathrm{r}} / S R_{\mathrm{a}}\right)$ 年均值为 $52 \%$ 。

\section{3 讨论}

为了区分土壤呼吸组分并定量菌根呼吸在土壤 碳释放中的贡献, 本研究采用改进的深浅环网孔法, 通过设置深环和菌丝环，隔离根系并允许菌丝生 长。结果表明深环的嵌入阻止了根系和菌丝进入, 深环土壤中菌丝密度显著低于环外土壤, 而菌丝环 因为设置了允许菌丝进入的窗口, 其环内土壤的菌 丝密度显著高于环外土壤。以上结果说明菌丝环达 到了隔离根系而允许菌根真菌菌丝进入的预定目 标。虽然深环和菌丝环达到了隔离菌丝与根系的目 的，但是同时也阻止了土壤水分和能量的侧向交换， 结果表明, 不同类型的土壤呼吸环(浅环、深环和菌 丝环)的放置虽没有影响环内土壤温度，但导致深 环和菌丝环内的土壤含水量显著提高, 这可能由于 

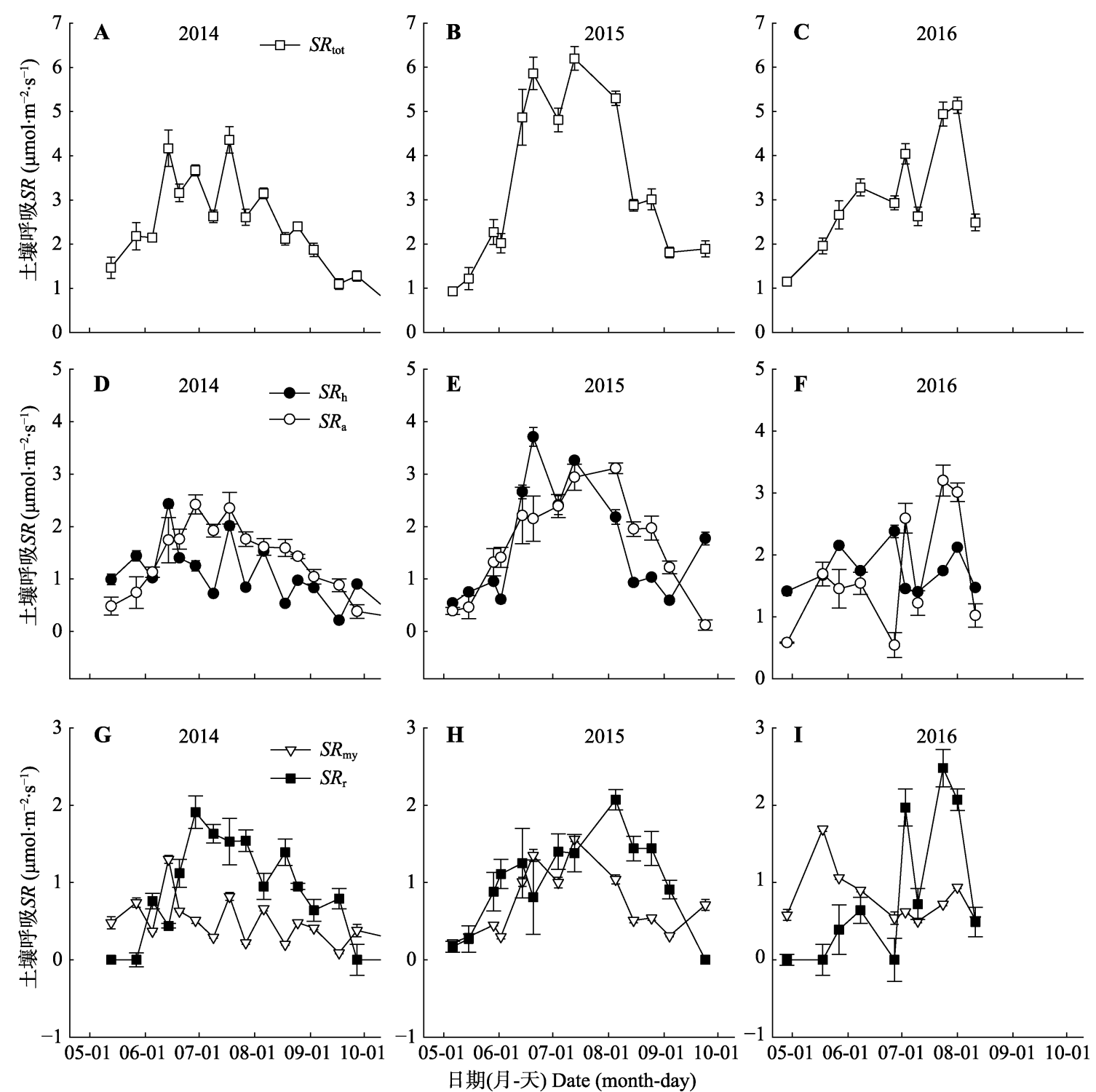

图8 2014-2016年生长季中不同土壤呼吸组分的季节变化(平均值标准误差)。SR $、 S R_{\mathrm{a}} 、 S R_{\mathrm{my}} 、 S R_{\mathrm{r}}$ 分别表示异养呼吸、自 养呼吸、菌根呼吸、根呼吸。

Fig. 8 Seasonal variations of different soil respiration components during growing seasons of 2014-2016 (mean $\pm S E)$. $S R_{\mathrm{h}}$, $S R$, $S R_{\mathrm{my}}$ and $S R_{\mathrm{r}}$ represent heterotrophic respiration, autotrophic respiration, mycorrhiza respiration and root respiration, respectively.

深环和菌丝环的嵌入隔断了植物的根系, 缺少了植 物水分的消耗，改变了环内土壤的水分状况，同时 缺少侧向水分交换使得深环和菌丝环内一直保持较 高的土壤水分状况。在半干旱草原地区, 水分和温 度是土壤呼吸的最主要限制因子。由于深环和菌丝 环内含水量的提高, 使得深环和菌丝环内的土壤呼 吸增强, 从而造成对异养呼吸和菌根呼吸组分的高 估。本研究中除2016年土壤呼吸与土壤含水量的关 系不显著外, 土壤呼吸及其组分与土壤温度和土壤 含水量均表现出显著的相关关系, 因此在深环和菌 丝环土壤呼吸的校正模型中同时考虑了土壤温度和 土壤含水量两个因子。模型的结果表明, 与仅考虑
单个因子相比，同时考虑温度和水分两个因子明显 提高了模型的解释度。通过建立土壤呼吸测量值与 土壤温度和水分拟和方程, 采用浅环内土壤温度和 水分测量值重新计算深环和菌丝环土壤呼吸。校正 后深环和菌丝环土壤呼吸速率比测量值分别下降了 $27 \%$ 和 $21 \%$ 。采用校正值计算出土壤呼吸各个呼吸 组分所占比例。

通过2014-2016三个生长季的连续测定，表明 内蒙古半干旱草原异养呼吸占土壤总呼吸的比例为 $45 \%-54 \%$, 根呼吸占土壤总呼吸的 $20 \%-34 \%$, 菌根 呼吸占土壤总呼吸的 $21 \%-26 \%$ 。与国内外研究(表2) 相比, 这和Heinemeyer等(2012)的研究结果是相似 www.plant-ecology.com 

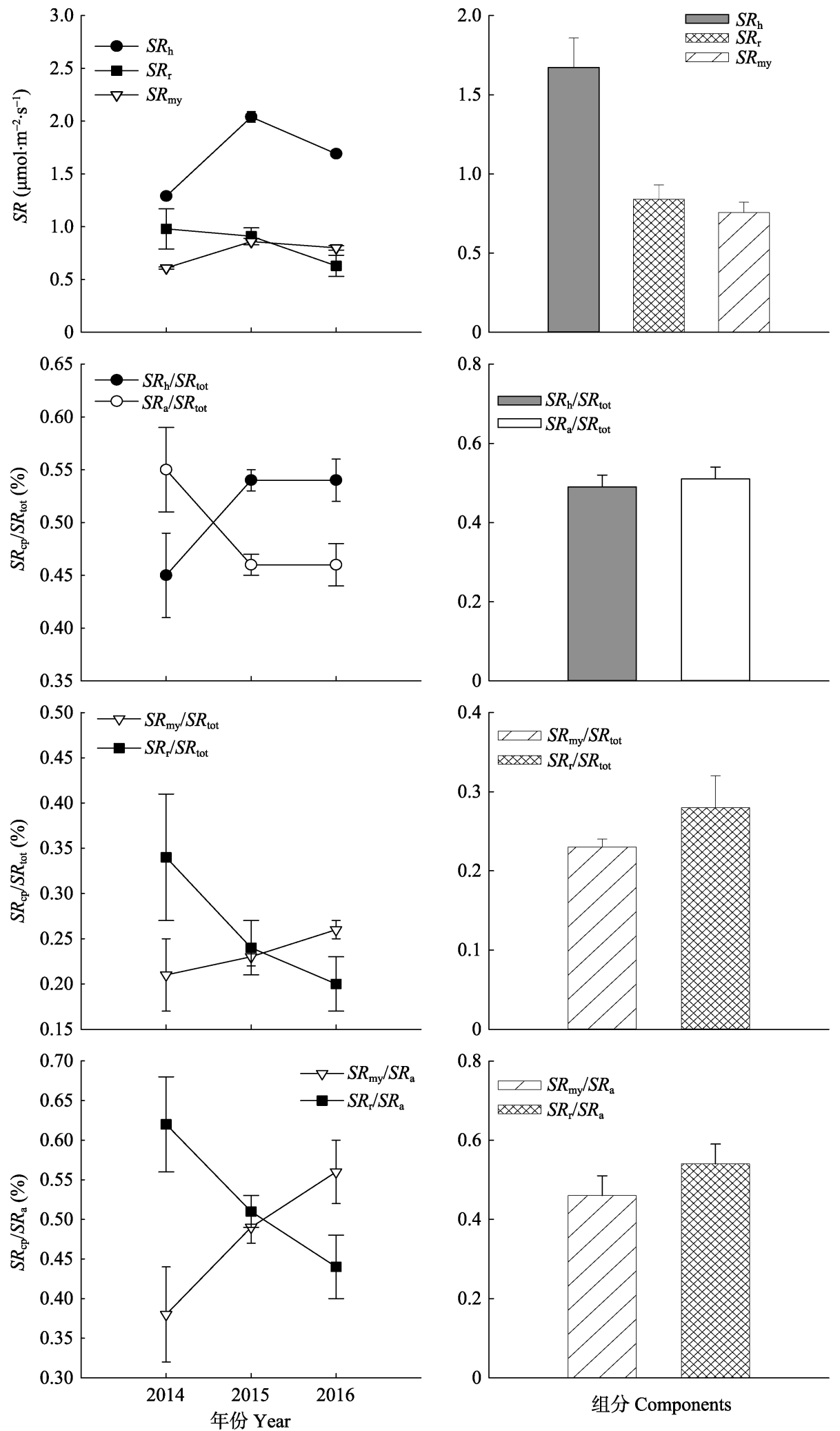

图9土壤呼吸(SR)及其各组分的年际变化(2014-2016)和3年平均值(2014-2016年5-8月份)以及土壤呼吸各组分占总呼吸的比 例和菌根呼吸、根呼吸分别占自养呼吸的比例的年际变化和3年平均值(2014-2016年5-8月份)(平均值土标准误差)。 $S R_{\mathrm{h}} 、 S R_{\mathrm{a}}$ 、 $S R_{\mathrm{my}} 、 S R_{\mathrm{r}} 、 S R_{\mathrm{tot}}$ 分别表示异养呼吸、自养呼吸、菌根呼吸、根呼吸和总呼吸。

Fig. 9 Interannual variations of soil respiration $(S R)$ and its components, the contribution of soil respiration components to total soil respiration and the contribution of $S R_{\mathrm{my}}$ and $S R_{\mathrm{r}}$ to $S R_{\mathrm{a}}$ during 2014-2016 and averages from May to August of the 3 years (mean $\pm S E) . S R_{\mathrm{h}}, S R_{\mathrm{a}}, S R_{\mathrm{my}}, S R_{\mathrm{r}}$ and $S R_{\mathrm{tot}}$ represent heterotrophic respiration, autotrophic respiration, mycorrhiza respiration, root respiration and total soil respiration, respectively. 
表2 菌根呼吸组分对土壤总呼吸贡献比例的相关研究结果

Table 2 Summary of studies on the contribution of mycorrhizal respiration to total soil respiration

\begin{tabular}{|c|c|c|c|c|c|}
\hline $\begin{array}{l}\text { 生态系统 } \\
\text { Ecosystems }\end{array}$ & $\begin{array}{l}\text { 地点 } \\
\text { Location }\end{array}$ & $\begin{array}{l}\text { 优势物种 } \\
\text { Dominant species }\end{array}$ & $\begin{array}{l}\text { 方法 } \\
\text { Methods }\end{array}$ & $\begin{array}{l}\text { 菌根呼吸贡献率 } \\
\text { Mycorrhizal contribution (\%) }\end{array}$ & $\begin{array}{l}\text { 文献来源 } \\
\text { Sources }\end{array}$ \\
\hline \multirow[t]{6}{*}{$\begin{array}{l}\text { 森林 } \\
\text { Forest }\end{array}$} & $\begin{array}{l}\text { 德国东南部 } \\
\text { Southeastern Germany }\end{array}$ & $\begin{array}{l}\text { 欧洲云杉 } \\
\text { Picea abies }\end{array}$ & $\begin{array}{l}\text { 内生长网孔袋法 } \\
\text { In growth mesh bags } \\
(2 \mathrm{~mm} \text { and } 45 \mu \mathrm{m})\end{array}$ & $18 \%-44 \%$ & $\begin{array}{l}\text { Neumann \& } \\
\text { Matzner, } 2014\end{array}$ \\
\hline & $\begin{array}{l}\text { 英国约克南部 } \\
\text { South of York, } \\
\text { UK } \\
53.91^{\circ} \mathrm{N}, 1.00^{\circ} \mathrm{W}\end{array}$ & $\begin{array}{l}\text { 美国黑松 } \\
\text { Pinus contortaDouglas } \\
\text { ex Loudon } \\
\text { 垂枝桦 Betula pendula }\end{array}$ & $\begin{array}{l}\text { 菌根网孔环设计 } \\
\text { Mycorrhizal mesh collar } \\
\text { design }(41 \mu \mathrm{m})\end{array}$ & $25 \%$ & $\begin{array}{l}\text { Heinemeyer et al., } \\
2007\end{array}$ \\
\hline & $\begin{array}{l}\text { 美国 } \\
\text { 宾夕法尼亚州 } \\
\text { Pennsylvania, USA }\end{array}$ & $\begin{array}{l}\text { 西黄松 } \\
\text { Pinus ponderosa }\end{array}$ & $\begin{array}{l}\text { 根模拟系统和同位素方法 } \\
\text { Root-mycocosm } \\
\text { and isotopic method }\end{array}$ & $19.4 \%$ & $\begin{array}{l}\text { Rygiewicz \& } \\
\text { Andersen, } 1994\end{array}$ \\
\hline & $\begin{array}{l}\text { 巴拿马共和国 } \\
\text { The Republic of Panama }\end{array}$ & Pseudobombax septenatum & $\begin{array}{l}\text { 内生长模拟系统 } \\
\text { In-growth mesocosms }\end{array}$ & $(14 \pm 6) \%$ & $\begin{array}{l}\text { Nottingham et al., } \\
2010\end{array}$ \\
\hline & $\begin{array}{l}\text { 德国中部 } \\
\text { Central Germany } \\
51.08^{\circ} \mathrm{N}, 10.45^{\circ} \mathrm{E}\end{array}$ & $\begin{array}{l}\text { Fagus sylvatica } \\
\text { 欧洲云杉 Picea abies }\end{array}$ & $\begin{array}{l}\text { 应用不同规格孔径的 } \\
\text { 尼龙网袋土钻法 } \\
\text { Soil cors using nylon mesh } \\
\text { bags of } 35 \text { and } 1 \mu \mathrm{m} \text { pore size }\end{array}$ & $3 \%, 8 \%$ & Moyano et al., 2008 \\
\hline & $\begin{array}{l}\text { 美国密歇根霍顿 } \\
\text { Houghton, Michigan, USA } \\
47.12^{\circ} \mathrm{N}, 88.55^{\circ} \mathrm{W}\end{array}$ & $\begin{array}{l}\text { 北美乔松 } \\
\text { Pinus strobus, } \\
\text { Quercus rubra }\end{array}$ & $\begin{array}{l}\text { PVC环和模型 } \\
\text { PVC collar and models }\end{array}$ & $28 \%$ & Lilleskov, 2017 \\
\hline \multirow[t]{2}{*}{$\begin{array}{l}\text { 草原 } \\
\text { Grassland }\end{array}$} & $\begin{array}{l}\text { 匈牙利 } \\
\text { Hungary } \\
46.69^{\circ} \mathrm{N}, 19.60^{\circ} \mathrm{E}\end{array}$ & $\begin{array}{l}\text { Festuca pseudovina, } \\
\text { Carex stenophylla, } \\
\text { 狗牙根 Cynodon dactylon }\end{array}$ & $\begin{array}{l}\text { 物理分离 }(40 \mu \mathrm{m} \text { 不锈钢网)和 } \\
\text { 同位素方法 } \\
\text { Physical separation: }(40 \mu \mathrm{m} \\
\text { inox mesh) and isotopic method }\end{array}$ & $(13 \pm 8) \%$ & Balogh et al., 2016 \\
\hline & $\begin{array}{l}\text { 英国约克郡北部 } \\
\text { North Yorkshire, UK } \\
54.00^{\circ} \mathrm{N}, 1.18^{\circ} \mathrm{W}\end{array}$ & 线毛草 Holcus lanatus & $\begin{array}{l}\text { 网孔环嵌插法 } \\
\text { Meshcollar insertion }(20 \mu \mathrm{m})\end{array}$ & $27 \%$ & $\begin{array}{l}\text { Heinemeyer et al., } \\
2012\end{array}$ \\
\hline \multirow[t]{2}{*}{$\begin{array}{l}\text { 农田 } \\
\text { Farmland }\end{array}$} & $\begin{array}{l}\text { 意大利北部 } \\
\text { Northern Italy } \\
47.30^{\circ} \mathrm{N}, 11.58^{\circ} \mathrm{E}\end{array}$ & Malus domestica & $\begin{array}{l}\text { 尼龙膜结合同位素法 } \\
\text { Polyester membrane }(5 \mu \mathrm{m} \text { and } \\
50 \mu \mathrm{m}) \text { with isotopic method }\end{array}$ & $11.6 \%$ & Tomè et al., 2016 \\
\hline & $\begin{array}{l}\text { 德国图林根 } \\
\text { Thuringia, Germany } \\
51.10^{\circ} \mathrm{N}, 11.77^{\circ} \mathrm{E}\end{array}$ & $\begin{array}{l}\text { 大麦 } \\
\text { Hordeum vulgare }\end{array}$ & $\begin{array}{l}\text { 尼龙网孔袋法 } \\
\text { Nylon mesh bags } \\
(1 \mu \mathrm{m} \text { and } 35 \mu \mathrm{m})\end{array}$ & $25.3 \%$ & Moyano et al., 2007 \\
\hline
\end{tabular}

的, 他们在温带草原, 利用相似的研究方法得到根 呼吸对土壤总呼吸的贡献是 $10 \%-30 \%$ ，菌根呼吸对 土壤总呼吸的贡献是 $27 \%$ 。而Balogh等(2016)研究了 干旱草原在干旱条件下, 菌根呼吸对土壤总呼吸的 贡献是(13 \pm 8$) \%$ 。Grimoldi等(2006)利用稳定同位素 技术, 发现多年生黑麦草的根被AMF侵染后, 土壤 中的 $\mathrm{CO}_{2}$ 通量增加了 $16 \%$ 。在森林生态系统中, Neumann和Matzner (2014)通过内生长网袋法发现, 在生长季菌根呼吸对土壤呼吸的贡献是 $18 \%-44 \%$ 。

Heinemeyer等(2007)和Lilleskov (2017)通过改进的 深浅环法发现, 菌根呼吸对土壤呼吸的贡献分别是 25\%和28\%。利用分室系统隔离装置, Rygiewicz和 Andersen (1994)及Nottingham等(2010)表明菌根呼 吸对土壤总呼吸的贡献分别为 $19.4 \%$ 和 $(14 \pm 6) \%$ 。 在农田中, Tomè等(2016)在苹果园利用同位素技术 发现菌根呼吸的贡献是 $11.6 \%$, Moyano等(2007)通 过尼龙网袋法发现菌根呼吸的贡献是 $25.3 \%$ 。这些 不同的结果可能与实验过程中不同的菌根和细根生
物量有关, 而这些是受土壤因素所影响的, 比如土 壤水分(Tedersoo et al., 2014), 土壤温度(Tomè et al., 2016), 可利用的营养(Koorem et al., 2014; Lauber et al., 2008)。已有的这些研究表明在不同的生境, 不 同的实验方法有关菌根呼吸对总呼吸的贡献估计有 所不同, 而对于半干旱草原此方面的研究较少需要 进一步的探索。

综上所述, 本研究在内蒙古半干旱草原区分土 壤呼吸组分所采用的深浅环网孔法，操作简便，资 金投入少，且是原位研究，对土壤的干扰相对较小, 很适合野外研究实验。并且3年较长时间的土壤呼吸 组分的分离研究, 为各个组分对土壤呼吸的贡献提 供了一个较为准确的范围, 异养呼吸对总呼吸的贡 献比例为 $51 \%$, 根呼吸的贡献比例为 $26 \%$, 菌根呼 吸的贡献比例为 $23 \%$ 。与国内外研究相比, 由于不 同的生态系统类型，不同的研究方法，菌根呼吸对 土壤呼吸的贡献有一定的差异性, 但是本研究能够 将菌根呼吸对土壤呼吸的贡献限定在一个相对准确 www.plant-ecology.com 
的范围内, 有助于我们更好地理解草原地下碳循环 过程，并预测其对未来气候的响应。

致谢 感谢中国科学院植物研究所孙秀峰老师在实 验工作中给予的指导。

\section{参考文献}

Andrews JA, Harrison KG, Matamala R, Schlesinger WH (1999). Separation of root respiration from total soil respiration using carbon-13 labelling during free-air carbon dioxide enrichment (FACE). Soil Science Society of America Journal, 63, 1429-1435.

Balogh J, Papp M, Pinter K, Foti S, Posta K, Eugster W, Nagy $\mathrm{Z}$ (2016). Autotrophic component of soil respiration is repressed by drought more than the heterotrophic one in dry grasslands. Biogeosciences, 13, 5171-5182.

Bhupinderipal-Singh, Nordgren A, Ottosson-Löfvenius M, Högberg MN, Mellander PE, Högberg P (2003). Tree root and soil heterotrophic respiration as revealed by girdling of boreal Scots pine forest: Extending observations beyond the first year. Plant, Cell \& Environment, 26, 1287-1296.

Cavagnaro TR, Smith FA, Smith SE, Jakobsen I (2005). Functional diversity in arbuscular mycorrhizas: Exploitation of soil patches with different phosphate enrichment differs among fungal species. Plant, Cell \& Environment, 28, 642-650.

Drigo B, Pijl AS, Duyts H, Kielak AM, Gamper HA, Houtekamer MJ, Boschker HTS, Bodelier PLE, Whiteley AS, Veen JAV (2010). Shifting carbon flow from roots into associated microbial communities in response to elevated atmospheric $\mathrm{CO}_{2}$. Proceedings of the National Academy of Sciences of the United States of America, 107, 10938-10942.

Fisher FM, Gosz JR (1986). Effects of trenching on soil processes and properties in a New Mexico mixed-conifer forest. Biology and Fertility of Soils, 2, 35-42.

Friese CF, Allen MF (1991). The spread of VA mycorrhizal fungal hyphae in the soil: Inoculum types and external hyphal architecture. Mycologia, 83, 409-418.

Grimoldi AA, Kavanova M, Lattanzi FA, Schaufele R, Schnyder H (2006). Arbuscular mycorrhizal colonization on carbon economy in perennial ryegrass: Quantification by ${ }^{13} \mathrm{CO}_{2} /{ }^{12} \mathrm{CO}_{2}$ steady-state labelling and gas exchange. New Phytologist, 172, 544-553.

Hanson PJ, Edwards NT, Garten CT, Andrews JA (2000). Separating root and soil microbial contributions to soil respiration: A review of methods and observations. Biogeochemistry, 48, 115-146.

Heinemeyer A, Hartley IP, Evans SP, de La Fuente JAC, Ineson $\mathrm{P}$ (2007). Forest soil $\mathrm{CO}_{2}$ flux: Uncovering the contribution and environmental responses of ectomycorrhizas. Global Change Biology, 13, 1786-1797.

Heinemeyer A, Tortorella D, Petrovičová B, Gelsomino A (2012). Partitioning of soil $\mathrm{CO}_{2}$ flux components in a temperate grassland ecosystem. European Journal of Soil Science, 63, 249-260.

Heinemeyer A, Ineson P, Ostle N, Fitter AH (2006). Respiration of the external myceliumin the arbuscular mycorrhizal symbiosis shows strong dependence on recent photosynthates and acclimation to temperature. New Phytologist, 171, 159-170.

Högberg P, Nordgren A, Buchmann N, Taylor AFS, Ekblad A, Högberg MN, Nyberg G, Ottosson-Löfvenius M, Read DJ (2001). Large-scale forest girdling shows that current photosynthesis drives soil respiration. Nature, 411, 789-792.

Jakobsen I, Abbott LK, Robson AD (1992). External hyphae of vesicular arbuscular mycorrhizal fungi associated with Trifolium subterraneum L. 2. Hyphal transport of ${ }^{32} \mathrm{P}$ over defined distances. New Phytologist, 120, 509-516.

Jakobsen I, Rosendahl L (1990). Carbon flow into soil and external hyphae from roots of mycorrhizal cucumber plants. New Phytologist, 115, 77-83.

Johnson D, Leake JR, Read DJ (2001). Novel in-growth core system enables functional studies of grassland mycelial networks. New Phytologist, 152, 555-562.

Kernaghan G (2013). Functional diversity and resource partitioning in fungi associated with the fine feeder roots of forest trees. Symbiosis, 61, 113-123.

Koorem K, Gazol A, Öpik M, Moora M, Saks Ü, Uibopuu A, Sõber V, Zobel M (2014). Soil nutrient content influences the abundance of soil microbes but not plant biomass at the small-scale. PLOS ONE, 9, e91998. DOI: 10.1371/journal.pone.0091998.

Kuzyakov Y, Larionova AA (2005). Root and rhizomicrobial respiration: A review of approaches to estimate respiration by autotrophic and heterotrophic organisms in soil. Journal of Plant Nutrition and Soil Science, 168, 503-520.

Lauber CL, Strickland MS, Bradford MA, Fierer N (2008). The influence of soil properties on the structure of bacterial and fungal communities across land-use types. Soil Biology \& Biochemistry, 40, 2407-2415.

Leake J, Johnson D, Donnelly D, Muckle G, Boddy L, Read D (2004). Networks of power and influence: The role of mycorrhizal mycelium in controlling plant communities and agroecosystem functioning. Canadian Journal of Botany, 82, 1016-45.

Lee MS, Nakane K, Nakatsubo T, Koizumi H (2003). Seasonal changes in the contribution of root respiration to total soil respiration in a cool-temperate deciduous forest. Plant and Soil, 255, 311-318.

Li YF, Gao R, Li Y, Qiu J, Cheng GS, Yin YF (2008). In vitro root respirations of different diameter classes for Chinese firs in relation to root parameters. Journal of Subtropical Resources \& Environment, 3(2), 19-24.

Lilleskov EA (2017). How does temperature affect forest "fungus breath"? Diurnal non-exponential temperaturerespiration relationship, and possible longer-term acclimation in fungal sporocarps. Fungal Ecology, 27, 24-35.

Luo YQ, Zhou XH (2006). Soil Respiration and the Environment. 
Higher Education Press, Beijing. 159-160. [骆亦其, 周旭 辉 (2006). 土壤呼吸与环境. 高等教育出版社, 北京. 159-160.]

Ma WH, Yang YH, He JS, Zeng H, Fang JY (2008). The relationship between the biomass and environmental factors in Inner Mongolia. Chinese Science C Series: Life Science, 38，84-92. [马文红, 杨元合, 贺金生, 曾辉, 方精云 (2008). 内蒙古温带草地生物量及其与环境因子的关系. 中国科学 C辑: 生命科学, 38, 84-92.]

Miller RM, Jastrow JD, Reinhardt DR (1995). External hyphal production of vesicular-arbuscular mycorrhizal fungi in pasture and tallgrass prairie communities. Oecologia, 103, $17-23$.

Moyano FE, Kutsch WL, Rebmann C (2008). Soil respiration fluxes in relation to photosynthetic activity in broad-leaf and needle-leaf forest stands. Agricultural and Forest Meteorology, 148, 135-143.

Moyano FE, Kutsch WL, Schultze ED (2007). Response of mycorrhizal, rhizosphere and soil basal respiration to temperature and photosynthesis in a barley field. Soil Biology \& Biochemistry, 39, 843-853.

Neumann J, Matzner E (2014). Contribution of newly grown extramatrical ectomycorrhizal mycelium and fine roots to soil respiration in a young Norway spruce site. Plant and Soil, 378, 73-82.

Nottingham AT, Turner BL, Winter K, van der Heijden MG, Tanner EV (2010). Arbuscular mycorrhizal mycelial respiration in a moist tropical forest. New Phytologist, 186, 957-967.

Rakonczay Z, Seiler JR, Kelting DL (1997). Carbon efflux rates of fine roots of three tree species decline shortly after excision. Environmental and Experimental Botany, 38, 243-249.

Rillig MC, Field CB, Allen MF (1999). Soil biota responses to long-term atmospheric $\mathrm{CO}_{2}$ enrichment in two California annual grasslands. Oecologia, 119, 572-577.

Rodeghiero M, Cescatti A (2006). Indirect partitioning of soil respiration in a series of evergreen forest ecosystems. Plant and Soil, 284, 7-22.

Rygiewicz PT, Andersen CP (1994). Mycorrhizae alter quality and quantity of carbon allocated below ground. Nature, $369,58-60$.

Saiz G, Black K, Reidy B, Lopez S, Farrell EP (2007). Assessment of soil $\mathrm{CO}_{2}$ efflux and its components using a processbased model in a young temperate forest site. Geoderma, 139, 79-89.

Shi JJ, Geng YB (2014). Study on the distinguishing of root respiration from soil microbial respiration in a Leymus chinensis steppe in Inner Mongolia, China. Environmental Science, 35, 341-347.

Slavíková R, Püschel D, Janoušková M, Hujslová M, Konvalinková $T$, Gryndlerová $H$, Gryndler $M$, Weiser $M$, Jansa $\mathrm{J}$ (2017). Monitoring $\mathrm{CO}_{2}$ emissions to gain a dynamic view of carbon allocation to arbuscular mycorrhizal fungi. Mycorrhiza, 27, 35-51.

Smith SE, Read DJ (2008). Mycorrhizal Symbiosis. Academic Press, London.

Talbot JM, Allison SD, Treseder KK (2008). Decomposers in disguise: Mycorrhizal fungi as regulators of soil C dynamics in ecosystems under global change. Functional Ecology, 22, 955-963.

Tedersoo L, Bahram M, Põlme S, Kõljalg U, Yorou NS, Wijesundera R, Ruiz LV, Vasco-Palacios AM, Thu PQ, Suija A, Smith ME, Sharp C, Saluveer E, Saitta A, Rosas M, Riit T, Ratkowsky D, Pritsch K, Põldmaa K, Piepenbring M, Phosri C, Peterson M, Parts K, Pärtel K, Otsing E, Nouhra E, Njouonkou AL, Nilsson RH, Morgado LN, Mayor J, May TW, Majuakim L, Lodge DJ, Lee SS, Larsson KH, Kohout P, Hosaka K, Hiiesalu I, Henkel TW, Harend H, Guo L, Greslebin A, Grelet G, Geml J, Gates G, Dunstan W, Dunk C, Drenkhan R, Dearnaley J, Kesel AD, Dang T, Chen X, Buegger F, Brearley FQ, Bonito G, Anslan S, Abell S, Abarenkov K (2014). Global diversity and geography of soil fungi. Science, 346, 1-11.

Tomè E, Ventura M, Folegot S, Zanotelli D, Montagnani L, Mimmo T, Tonon G, Tagliavini M, Scandellari F (2016). Mycorrhizal contribution to soil respiration in an apple orchard. Applied Soil Ecology, 101, 165-173.

Wang C, Yang J (2007). Rhizospheric and heterotrophic components of soil respiration in six Chinese temperate forests. Global Change Biology, 13, 123-131.

Wilson GWT, Rice CW, Rillig MC, Springer A, Hartnett DC (2009). Soil aggregation and carbon sequestration are tightly correlated with the abundance of arbuscular mycorrhizal fungi: Results from long-term field experiments. Ecology Letters, 12, 452-461.

Xu M, DeBiase TA, Qi Y, Goldstein A, Liu Z (2001). Ecosystem respiration in a young ponderosa pine plantation in the Sierra Nevada Mountains, California. Tree Physiology, 21, 309-318.

Yan L, Chen S, Huang J, Lin G (2010). Differential responses of auto- and heterotrophic soil respiration to water and nitrogen addition in a semiarid temperate steppe. Global Change Biology, 16, 2345-2357.

Zhang Q, Lei HM, Yang DW (2013). Seasonal variations in soil respiration, heterotrophic respiration and autotrophic respiration of a wheat and maize rotation cropland in the North China Plain. Agricultural \& Forest Meteorology, $180,34-43$.

Zhu JJ, Xu H, Xu ML, Kang HZ (2003). Review on the ecological relationships between forest trees and ectomycorrhizal fungi. Chinese Journal of Ecology, 22(6), 70-76. [朱教君, 徐慧, 许美玲, 康宏樟 (2003). 外生菌根菌与 森林树木的相互关系. 生态学杂志, 22(6), 70-76.]

责任编委: 朱 彪 责任编辑: 李 敏 